\title{
HYBRID ITERATIVE SYSTEM SPECIFICATION OF CYBERPHYSICAL SYSTEMS: NEUROCOGNITIVE BEHAVIOR APPLICATION
}

\author{
Bernard P. Zeigler \\ University of Arizona \\ RTSync Corporation \\ 12500 Park Potomac Ave. \\ Potomac, MD, 20854, USA \\ zeigler@rtsync.com
}

\begin{abstract}
This paper extends the theory of iterative systems specification for application to a class of cyber-physical systems. We employ the finite state regular language framework as a basis for models of neuronal structure and behavior aimed at closing the gap between circuits and cognitive behavior. Although the biological brain is rarely considered in the context of cyber-physical systems, we draw insight from parallels between the physical and cognitive faculties of humans with those of such engineered systems. We show how hybrid iterative systems specification offers an abstraction that is more fundamental than DEVS that supports development of simulation models of cyber-physical systems. Computational models of dynamic brain activity are needed to understand the complex patterns of signaling and communication within and between brain networks. This paper sets up a theoretical framework for future research and development of brain and other cyber-physical models.
\end{abstract}

Keywords: cyber-physical systems, DEVS, hybrid system, iterative system specification, neurocognition modeling

\section{INTRODUCTION}

Cyber-physical systems (CPS) are "smart" systems that include engineered interacting networks of physical and computational components including sensing, computation, and actuation. Development of such systems through the fundamentals of Systems Theory (Wymore 1967) supported by an equally robust modeling and simulation (M\&S) theory is the preferred way forward (Mittal and Tolk 2020). From a systems theoretic perspective, a CPS is a hybrid system composed of both continuous and discrete component systems. Traditionally, a continuous dynamic system operates in continuous time with input, state, and output as real-valued vectors while discrete dynamic systems include both discrete event and discrete time systems. At the fundamental level, there are various ways to model both timed and untimed systems, all of which can be transformed to, and studied within, the formal Discrete Event Systems (DEVS) theory (Mittal and Zeigler 2017; Mittal and Risco-Martin 2016; Vangheluwe 2000).

Iterative system specification (ISS) is intended to be an abstraction that provides an approach to hybrid M\&S at the transdisciplinary level (Zeigler, Muzy, and Kofman 2018). This abstraction is more fundamental than DEVS in that it serves as a precursor that provides representations for the preimages of reality that are eventually presented as crisp discrete events. In fact, DEVS has abstracted away the original squishy boundaries begging the question of how can one come up with the events, states, and components in the first place. ISS helps restore more of the left out reality. This provides a more nuanced approach to hybrid M\&S at a fundamental systems level that can then be implementable in the computational medium 
of DEVS. In other words, ISS offers a constructive modeling approach to general systems development. Muzy et al (2018) employed ISS as the main structure for computational modeling of dynamic systems with memory. They formally mapped spiking neuronal models to ISS structures using discrete-event segmentation obtained by a combination based on event counting, time intervals and event detection. Zeigler and Muzy (2017) gave definitions for single and collective neural computation based on the DEVS formalism that fundamentally include temporal and probabilistic characteristics of neuronal system inputs, state, and outputs.

\subsection{The brain as a hybrid system modeled by ISS}

Although the biological brain is rarely considered in the context of CPS, we can draw insight from parallels between the physical and cognitive faculties of humans with those of such engineered systems (Castro et al 2019). Despite growing scientific interest, we know little about how the brain is organized into circuits that function as individual processors and the language that they use to communicate and interact with sensors and effectors (Makin 2016). Neuroscience seeks to understand how such circuits lead to perception, thought, and action. However, currently, the gap between circuits and behavior is too wide to bridge (Carandini 2012). Neural computations that occur in individual and populations of neurons offer an intermediate level to bridge the gap. Computation supplies a common language for neural and cognitive researchers. Here we show that iterative System Specification (ISS) modeling (Zeigler, Muzy, and Kofman 2018) can support linking the language of the brain with high level cognition and low level sensing and actuation. Bridging the gap requires focusing on a cognitive domain of interest and showing how the functions involved can be, through stages of hierarchical decomposition, eventually implemented in neural "wet-ware." In our current state of knowledge, development of the successive stages must realistically be driven by abstractions and simplifications that enable model construction that can be related both to plausible biological constraints as well as observed data gathered at higher levels.

To illustrate how ISS modeling applies to bridging the neural-cognitive gap, let's consider greetings interaction. At the cognitive level, greetings are exchanged through natural language for which much is known about its syntax, semantics, and pragmatics. In the hierarchy of specification framework, this is at the I/O Behavior level and we need to climb to the I/O Function and State System levels to get more explanatory, mechanistic, and eventually predictive capability. Finally, at the coupled systems level we come to neural networks that can eventually yield understanding of the language of the brain that mediates the exchanges of greetings in natural language. Although available natural language computational frameworks deal with the complexities of fully expressive conversations, here by design, we focus on the simplest of such interactions. Accordingly, we need a basis that is adequate for expressing greetings but also has potential for scaling up to more complex conversations.

In this paper, we identify the finite state regular language framework as such a basis. In brief, regular expressions are strings of symbols that include the alphabet and connectors for concatenation, Boolean operations, and Kleene star (denoting iterative concatenation) (Yu (1997). We will show how iterative systems specification offers an abstraction that supports development of hybrid cyber-physical models. To show this we prove that given a temporally represented finite alphabet and a regular expression over the alphabet, we can construct an iterative systems specification coupled model that constitutes a networked recognizer for the regular expression. Based on this construction, we will discuss how to close the gap between cognitive behavior and neural circuits.

Regular expressions are descriptions of behavior at the I/O Behavior level for which there are computable realizations by finite state automata. Indeed, for every regular expression there is a finite state acceptor (that can recognize strings in its language) and a finite state generator that can generate such strings (McNaughton 1960). Greetings can then be modeled as involving strings generated and recognized by such finite state systems. Therefore, we take this framework as the basis for the computations that mediate between cognitive and neural domains. However to connect with the latter, we need to identify realizations of such system specifications at the coupled system level involving neural componentry and potentially other needed cellular elements. 
One possibility for transition to the coupled model level is to recognize a regular expression as a hierarchical construction based on successive application of its operators. For instance, "grumpy $\|$ cheerful \& morning $\|$ cheerful \& noon" is composed of concatenated letters for words, combined by the conjunction (\&) and then by the disjunction $(\|)$. It could express a perception that leads to no response (if the encountered person appears to be grumpy) or either "good morning" or "good afternoon" (if s/he appears to be cheerful.) If we have atomic models for the letters and templates for composing with the operators then we can realize such expressions by hierarchical DEVS models. More precisely, recognizing that words are abstractions of patterns of sounds over time, we show how neural compositions can generate and recognize such temporal patterns using templates that can be expressed in plausible neural architectures. For example, spike density generators (where density or spike frequency could code for letters (Muzy et al 2018) as well as activation and input/output ports coupled by sequential and parallel coupling patterns.

In the next section we review ISS and then discuss DEVS Recognizer models before presenting results on DEVS network representation of regular languages. This sets the basis for extension of the results to continuous inputs and hybrid cyber-physical model construction. Finally, we come to application of the concepts to closing the gap between cognitive behavior and neural circuits.

\section{ITERATIVE SYSTEM SPECIFICATIONS}

Iterative system specification combines effective computation theory with systems theory in a way that allows us to specify systems in an event-like computational manner that is ultimately implementable in DEVS-based M\&S environments (Muzy et al 2018). A brief review of ISS concepts follows:

\subsection{Input Generators}

Input generators are relatively short time segments (functions of finite time intervals) that can be concatenated to form longer segments. For example, Figure 1(a) shows three types of generators: 1) null segments that can last any finite duration, 2) constant segments that can last any finite duration and 3) pulse segments lasting at most a duration T (e.g., 10 seconds). As illustrated in Figure 1(b), we consider composite inputs segments composed of concatenated generators placed one after another end-to-end. Such segments can be parsed into their component parts by a process called maximal length segmentation (MLS) (Zeigler, Muzy, and Kofman 2018). With input segments broken into generators we can generate the state trajectories associated with the system starting in its different states taking jumps over long time intervals as shown in Figure 1(c). To do this we need the transition function to be given in terms of the generators so that given a starting state and an input generator we compute a state at the end of the generator. Doing it this way can save a lot of computation that would otherwise be done with small steps as suggested in the figure. Of course, it might be necessary to do the small step computation to compile the state transition function for the generators. There is still much utility in doing this, since the compiled form can be used every time the generator appears in an input segment. Also if there is an analytic solution for the generator input's effect on the state then we can use that within the large step-based computation. Since the output trajectory is computed from that state trajectory, we can compute the output trajectories corresponding to the input generators as well. For these computations to produce effective results they must terminate. Conditions that guarantee such effective computation, called progressivity and well-definition, are discussed in (Zeigler, Muzy, and Kofman 2018), in particular, Theorem 12.1 shows how an ISS specifies a well-defined system by extending the single segment transition function to multi-segment inputs 


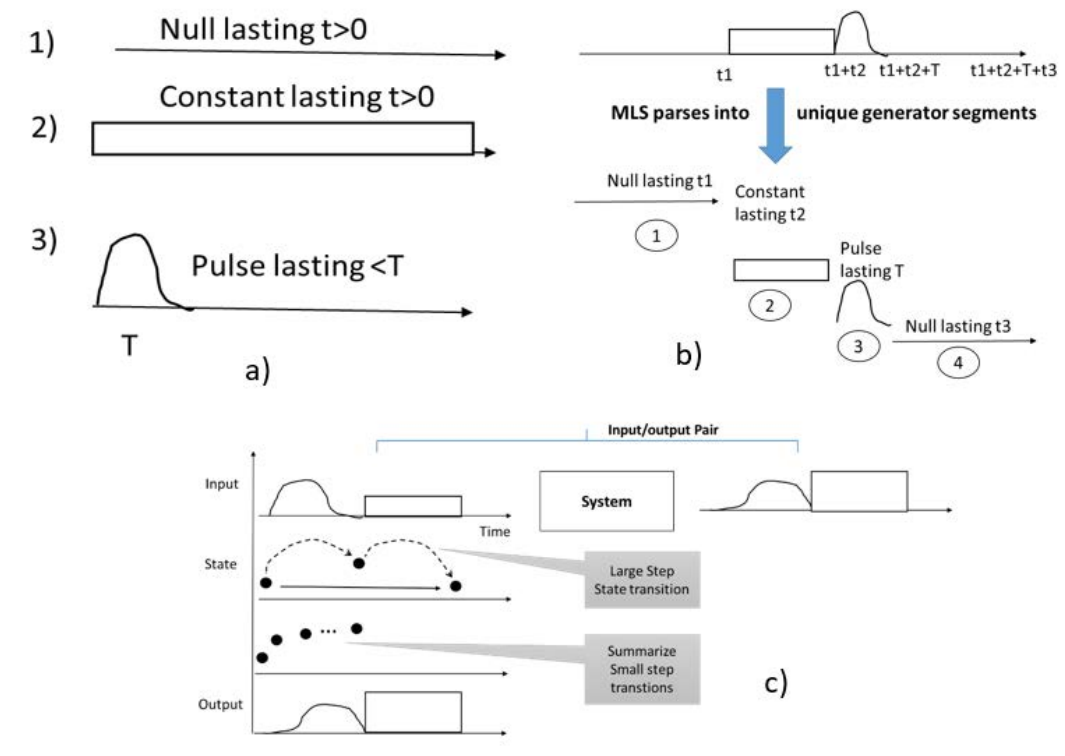

Figure 1: (a) Illustrating input generators, (b) Maximal Length Segmentation, (c) Computing with generators.

\subsection{Hybrid Iterative System Specifications}

Figure 2 maps out some of variants of ISS and places them in relation to each other and to the general system concept. Two relations are shown. One in which a formalism simulates another and the other in which one specifies another. DEVS simulates iterative systems specifications in the sense that given an iterative systems specification, there is a DEVS that can simulate it. Besides PDEVS (Parallel Discrete Event Systems Specification), several other iterative specifications are shown in Figure 2. Schedule Iterative Specification, introduces a time scheduling concept explicitly into the basic ISS. This allows the modeler to employ a time advance function that generalizes the time advance concept of PDEVS. It does not extend the class of systems that are specified since the modeler can already define such a function on a case-by-case basis to determine when an internal event should happen. In contrast, the time advance function in Schedule Iterative Specification provides this capability explicitly giving it a well-defined semantics. PDEVS then turns out to be a specific kind of Schedule Iterative Specification. A focus of this paper, Hybrid Iterative Specification is a further specialization of Schedule Iterative Specification that supports hybrid co-simulation (Zeigler, Muzy, and Kofman 2018). 


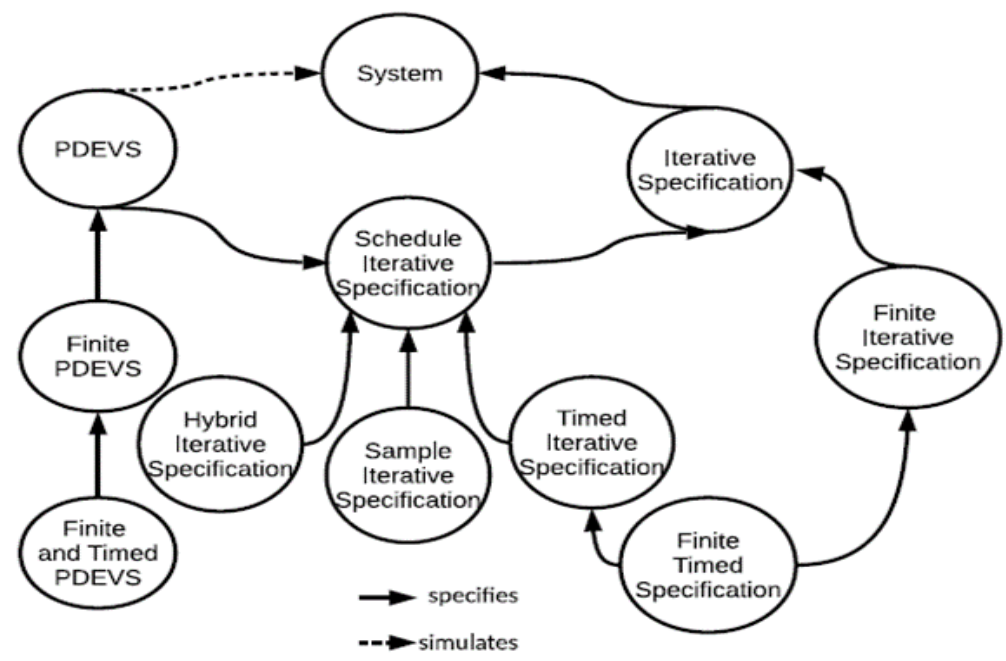

Figure 2: Class mapping of iterative system specifications.

\section{DEVS RECOGNIZER MODELS}

Let DEVS $(\mathrm{X})$ be a set of DEVS segments based on $\mathrm{X}$ a finite alphabet. Given an input segment, $\varphi$ ${ }_{\mathrm{t} 1}>\mathrm{X} 1 \varphi_{\mathrm{t} 2}>\mathrm{X} 2 \ldots \varphi_{\mathrm{tn}}>\mathrm{Xn}$ we strip the time information to obtain the string $\mathrm{x} 1, \mathrm{x} 2, \ldots, \mathrm{xn}$. The erasure of time information is called the natural encoding of DEVS segments to strings. We extend the concept of language recognition and generation from sequences to DEVS segments. Roughly, this requires that the DEVS model partitions the set of all segments into accepted and non-accepted sets just in case the stripped sequences are partitioned in the same manner by an automaton. Indeed, the way we proceed is to extend a finite state automaton to a DEVS in such a way that the language it accepts is in fact, the set of sequences stripped from the DEVS input segments. Figure 3 illustrates the approach. An atomic model that recognizes a single symbol e.g., the language $\{x\}$ appears in Fig. 1a) with input Activate to send it from the Wait state to the Hold State as shown in Fig 3c). After this, an input with label $\mathrm{x}$ happening some time later will cause the model to enter the Send state and to output an Activate output signaling the acceptance of the input. Any other input label will return the model to the Wait state without sending such an output. The model in DEVS set notation appears in Fig 3b).

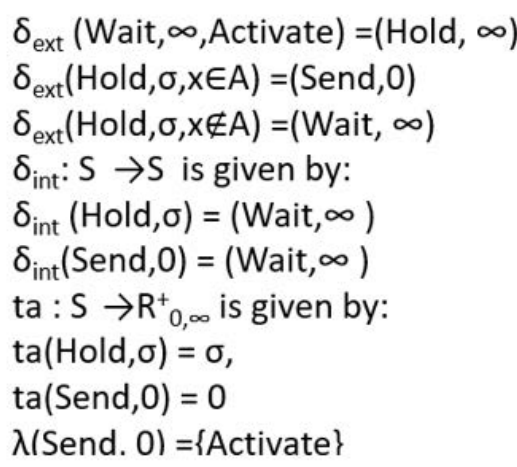

b)

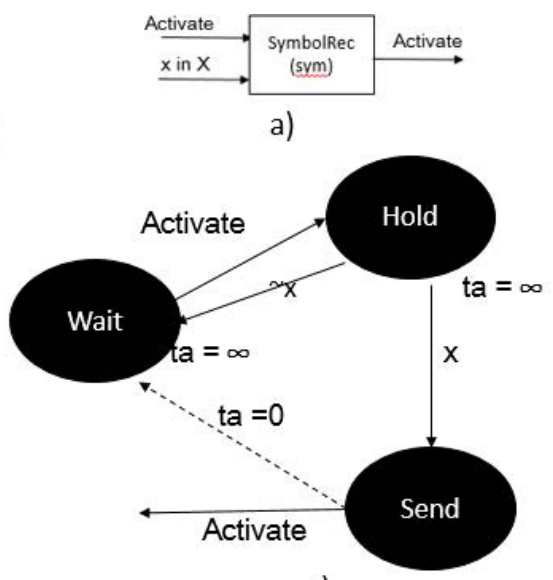

c)

Figure 3: DEVS Symbol Recognizer. 


\subsection{Constructing a DEVS Recognizer for segments sets in DEVS(X)}

As illustrated in Figure 4 a), we define an input-driven DEVS as a DEVS model for which the time advance in all states is infinite. Effectively, this means that the only source of state change is from external events and we may omit the internal transition function as in the definition: $\mathrm{M}=<\mathrm{X}, \mathrm{S}, \delta_{\text {ext }}, \mathrm{ta}>$ where $\operatorname{ta}(\mathrm{s})=\infty$, for all $\mathrm{s} \in \mathrm{S}$. For convenience we also omit the output function. Also we will require that the elapsed time, e plays no role in state transitions due to inputs so that: $\delta_{\text {ext }}(\mathrm{s}, \mathrm{e}, \mathrm{x})=\delta(\mathrm{s}, \mathrm{x})$. Now, as illustrated in Figure 4 b), a transition system, underlying a finite automaton, can be defined as $T=<X, S, \delta>$ where $\delta: S \times X \rightarrow S$. So we have Proposition 1.

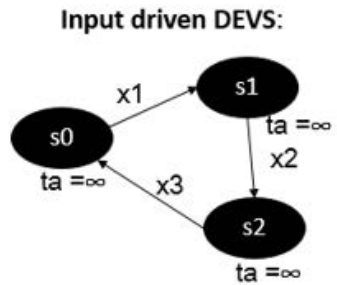

a)

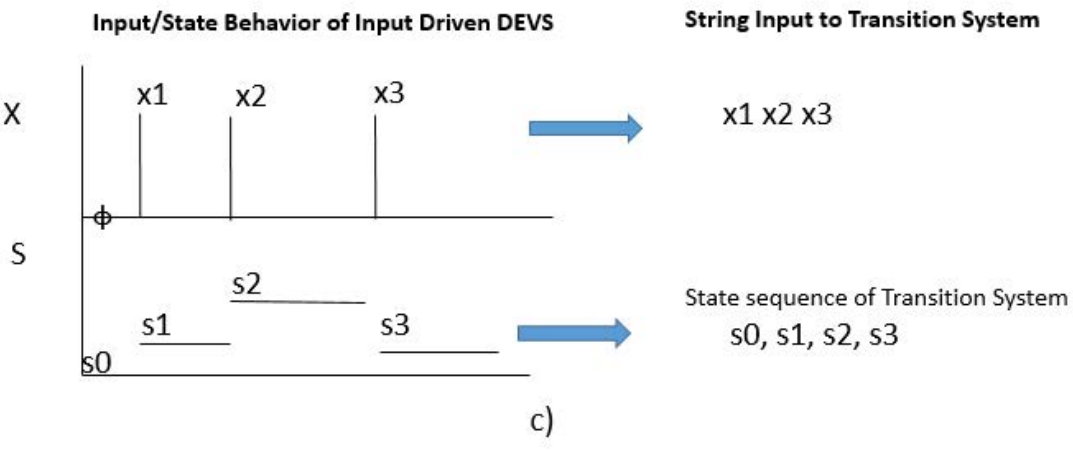

Figure 4: Mapping from input-driven DEVS to transition system.

Proposition 1. Given a transition system, T there is a DEVS M that homomorphically simulates it.

A sketch of the proof is illustrated in Figure $4 \mathrm{c}$ ). Construct a DEVS $\mathrm{M}$ whose states and transitions are labelled in one-one correspondence to those of T. Suppose that $\mathrm{T}$ undergoes a state sequence s0,s1,s2,s3 in response to an input sequence $\mathrm{x} 1, \mathrm{x} 2, \mathrm{x} 3$. Then if $\mathrm{M}$ is fed an event segment with natural encoding to $\mathrm{x} 1 \mathrm{x} 2 \mathrm{x} 3$, $M$ will undergo the state trajectory shown which in stripped form matches that of T. Technically, we can show that the natural encoding together with the one-one state mapping constitute a homomorphic simulation (see definition in Zeigler, Muzy, and Kofman (2018)) of T by M.

We also readily have the converse:

Proposition 2. Given an input-driven DEVS, there is a state transition system that it homomorphically simulates.

Now we can extend $\mathrm{T}$ to an automaton $\mathrm{A}_{\mathrm{T}}$ by adding to it labels for an initial state and a set of final states. Likewise, extend $M$ to a DEVS Recognizer, $R_{M}$ with addition of the same initial state and set of final state labels. Then stated in succinct form:

Corollary 1. The language accepted by $R_{M}$ is also accepted by $A_{T}$ 
Expanded, this means that the set of DEVS segments that drive the input free DEVS from its initial state to a final state, when stripped of their timing, is the set of sequences that drive the corresponding automaton from its initial state to one of its final states.

Note that to conform to the input/output interface for the DEVS model of Figure 1a), we have the model start in a Wait state and have it transition to the initial state upon receiving an Activate output. Likewise, we have it generate an Activate output when transitioning into a final state. This converts its input-free nature to a full-fledged DEVS. However, this interaction is only manifest for the Activate interface.

Theorem 1. A subset of DEVS $(X)$ is recognized by an input-driven DEVS if, and only if, the natural encoding of the set is a regular language. We call such a subset of DEVS $(X)$ a regular language of DEVS segments.

The proof centers on the fact that the internal operation of an input-driven DEVS is exactly replicated in the automaton constructed from it. Conversely if a set is accepted by an automaton then an input-free DEVS can be constructed that replicates this behavior in relation to the natural encoding.

\section{REGULAR REALIZATION AT THE DEVS NETWORK LEVEL}

In this section our goal is to develop templates for realizing the behavior expressed by regular languages in the form of DEVS coupled models. This will be done by realizing finite state automata in network form in a particular way that allows generalization to continuous time segments to be done in the next section.

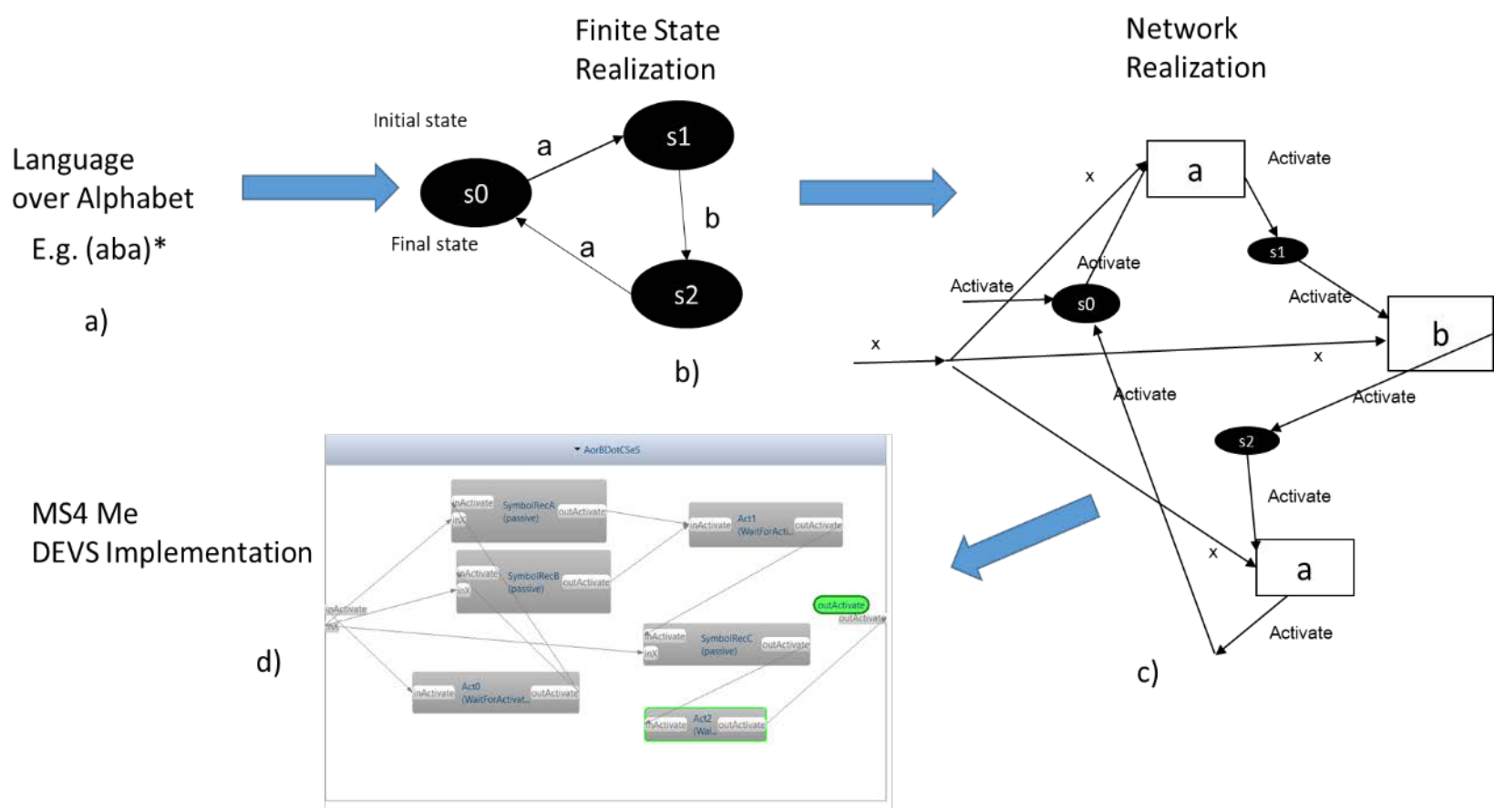

Figure 5: Realization of Regular language at Finite State and Network levels.

There are multiple representations of a regular language as a regular expression and as a finite state automaton. The mapping in Figures 5 a) and b) gives a simple example. Further there are multiple realizations of finite state machines in networked form as e.g., well-known clocked sequential circuits. As illustrated in Figures $5 \mathrm{~b}$ ) and c), the realization approach we take employs the recognizers for elementary symbols from Figure 1. In the mapping, each transition label is mapped to a recognizer of that label and each state is mapped to an activation node shown as a black oval component. For example, the states s0 and $\mathrm{s} 1$ are mapped to activation nodes $\mathrm{s} 0$ and $\mathrm{s} 1$, while the transition from $\mathrm{s} 0$ to $\mathrm{s} 1$ labelled a is mapped to a recognizer for a. Starting in initial state s0 is represented by external activation of its representative 
component. External input is coupled to each recognizer's input port. As in Figure 1, a recognizer can only pass on activation to its target state representative if has received activation from a predecessor state. The mapping is algorithmic and can be implemented to generate coupled model recognizers for the source regular expression as illustrated in Figure $5 \mathrm{~d}$ ) in a DEVS simulation environment (e.g MS4 Me (Seo et al 2013)). The activation node behavior can be easily captured by a simple atomic DEVS which produces an Activate output in response to the first Activate input that it receives. Formally, we can prove the

Theorem 2. A regular language of DEVS segments can be realized by a coupled model of symbol recognizers and activation nodes.

The proof shows that exactly one activation node is in the active state and it enables the recognizers for its corresponding state's outgoing input labels. Only one recognizer for each label accepts the next external event (assuming deterministic automata) and activates the target activation node representing the next automaton state. The proof then proceeds by induction to show that at the end of the input segment a final state is reached or not according to whether or not it is in the regular language.

\section{EXTENSION TO CONTINUOUS TIME SEGMENTS}

In the spirit of iterative system specifications (ISS), we say that a structure, $\mathrm{R}=\left\langle\Omega, \mathrm{Q}, \mathrm{q}_{0}, \delta, \mathrm{F}>\right.$ is a Recognizer where $\Omega$ is an admissible set of generators (continuous time segments such as in Figure 1 ), $\delta$ : $\mathrm{Q} \times \Omega \rightarrow \mathrm{Q}$ is a segment transition function with the properties of an ISS, and where $\mathrm{q}_{0} \in \mathrm{Q}$ is an initial state, and $\mathrm{F} \subseteq \mathrm{Q}$ is final set of states. The language recognized by $\mathrm{R}$ is $\mathrm{L}_{\mathrm{R}}=\left\{\omega \mid \delta^{+}\left(\mathrm{q}_{0}, \omega\right) \in \mathrm{F}\right\}$ i.e., the set of segments that take the initial state to a final state using the welldefined transition function, $\delta^{+}$extended from $\Omega$ to $\Omega^{+}$.

In what follows, we characterize a class of recognizers generalizing from DEVS segments and finite state automata with the extension to implementations closer to neural networks.

We show how to use to define a recognizer for an arbitrary segment, $\mu$. The idea is that we store $\mu$ in a register and compare any incoming segment, $\omega$ against it at each time instant until we run out of input. If there is any mismatch before then, we reject the input segment, otherwise it is accepted. Formally,

$\mathrm{R}(\mu)=<\Omega, \mathrm{Q}, \mathrm{q}_{0}, \delta, \mathrm{F}>$

where $\Omega=(\mathrm{X}, \mathrm{T})$ where $\mathrm{T}=[0, \infty]$,

$\mathrm{Q}=\{\mu\} \times \mathrm{X} \times \mathrm{X} \times \mathrm{T}$

where $\mathrm{q}=(\mu, \mathrm{x}, \mathrm{y}, \sigma)$ with $\mathrm{x}, \mathrm{y} \in \mathrm{X}$ and $\sigma \in \mathrm{T}$,

$\mathrm{q}_{0}=(\mu, \varphi, \varphi, \operatorname{len}(\mu))$,

$\mathrm{F}=\{(\mu, \mathrm{x}, \mathrm{y}, 0) \mid \mathrm{x}=\mathrm{y}\}$.

For any input segment $\omega$,

$\delta\left(\mu, \mathrm{x}, \mathrm{y}, \sigma, \omega_{\triangleright}\right)=(\mu, \mu(\mathrm{t}), \omega(\mathrm{t}), \sigma-\mathrm{t})$ if $\mu(\mathrm{t})=\omega(\mathrm{t})$ and $\mathrm{t} \in \operatorname{dom}(\mu)$

$=(\mu, \varphi, \varphi, \infty) \quad$ otherwise

Here, the initial state starts with $\mu$ in the register and a timer set to its length, len $(\mu)$. As time t proceeds, the left segment, $\omega_{\triangleright}$ of the input is read and its last value is stored for comparison to that of $\mu$. A mismatch results in transition to dead state $=(\mu, \varphi, \varphi, \infty)$. Acceptance occurs when reaching the end of test segment as indicated by the expiration of the timer $\sigma$ provided that no mismatch has occurred earlier. This construction is phrased as the

Proposition 3. For any segment, $\mu$ that is closed under left segmentation, there is a recognizer, $R(\mu)$.

The proof requires proving that the defined single step transition function satisfies the composition property which is not hard to do. 
Now, starting with a finite alphabet, A and a finite state automaton as in Figure 5 a) and b) we map the members to time segments $\Omega_{\mathrm{A}}=\left\{\mu_{\mathrm{a}} \mid \mathrm{a} \in \mathrm{A}\right\}$ as temporal representations such as sound patterns. Then the recognizers shown in Figure 5 c) take the form of recognizers for the individual segments $\mu_{\mathrm{a}}$. Further we have just shown that in principle, there is at least one recognizer system (in the form of an ISS) for each such segment. In fact we can extend each $\mathrm{R}\left(\mu_{\mathrm{a}}\right)=<\Omega, \mathrm{Q}, \mathrm{q}_{0}, \delta, \mathrm{F}>$ with the $\mathrm{I} / \mathrm{O}$ interface needed to substitute for the existing symbol recognizers. This requires the addition of a state to wait for an Activate input and generation of an Activate output when reaching a final state at the end of the test segment. This leads to the

Theorem 3. (Regular languages over symbols with temporal representations): Given a finite alphabet, $A$ with a set $\Omega_{A}=\left\{\mu_{a} \mid a \in A\right\}$ of temporal representations, and a regular expression over $A$, we can construct an ISS coupled model that constitutes a recognizer for the regular expression.

The proof requires defining a coupled model of $\mathrm{R}\left(\mu_{\mathrm{a}}\right)$ components and activation nodes following the pattern of Figure $5 \mathrm{c}$ ). Further the activation nodes can be defined as ISS atomic models similar to the ones already discussed. Then the proof shows that the coupled model so constructed is a valid coupled ISS model. It is simplified by the fact that only a small subset of non-interacting components is active at any time.

\section{MOVING TO CLOSE THE NEURO-COGNITION GAP}

The foregoing representation theorem places recognition networks in the middle of the gap between cognitive behavior and neural realization. One addition that is needed and close at hand is to perform a similar temporal realization of generators whose outputs are regular languages. We leave this for an extension of this paper to a journal format. A second extension is to broaden the class of recognizers of elementary time segments that can be components in the recognition networks. This should be driven by the goal of finding realizations that are biologically plausible. Since recognition involves partitioning into subclasses, we will show that an ideal general form of system specification to work is the hybrid iterative system specification (HISS) defined by Zeigler, Muzy, and Kofman (2018). In what follows, we shall formulate a neuron model within the HISS formalism along lines of the spiking neuronal models mapped to ISS structures by Muzy et al (2018) and Ciatto et al (2017). An HISS is given by:

$\mathrm{G}=(\Omega, \mathrm{Q}, \delta, \lambda, \mathrm{ts}, \mathrm{C}, \mathrm{A})$ where

$\Omega$ is an admissible set of generator segments

$\delta: \mathrm{Q} \times \Omega \rightarrow \mathrm{Q}$ is the single segment transition function, with the composition property

$\lambda: \mathrm{Q} \rightarrow \mathrm{Y}$ is the output function

ts $: \mathrm{Q} \rightarrow \mathrm{R}_{0,}^{+}$, is the schedule function, $\operatorname{ts}(\mathrm{q})=$ time to next internal event.

$\mathrm{C}: \mathrm{Q} \rightarrow$ Bool, the state event predicate

$\mathrm{A}: \mathrm{Q} \rightarrow \mathrm{Q}$, the action function

This type of ISS combines both time scheduled events with state events which are generated under conditions specified in $\mathrm{C}$ and result in instantaneous state change given by A (see (Zeigler, Muzy, and Kofman 2018) for details on how this specifies an ISS). A spiking neural model that illustrates this form of computational modeling considers $\Omega$ as a set of pulses as illustrated in Figure 1 a) 3. The model operates in two modes: charging and discharging. In the charging mode it integrates its input pulse using linear systems processing. In discharging mode it is refractory, not responding to external input. We present the model informally: A typical state $\mathrm{q}=(\mathrm{v}$, mode) where $\mathrm{v}$ is a real value that measures electric charge (actually membrane potential), and mode is a binary variable. The transition function is a linear systems response (Zadeh and Desoer 2008) to a pulse $\omega$, in charge mode, $\delta\left(\mathrm{v}\right.$, charge, $\left.\omega_{t}\right)=\left(\mathrm{e}^{\mathrm{At}} \mathrm{v}+\int \mathrm{e}^{\mathrm{A}(\mathrm{t}-\tau)} \mathrm{B} \omega_{\tau>} \mathrm{d} \tau\right.$, charge) $(\mathrm{A}, \mathrm{B}$ are negative and positive parameter values $)$ and in discharge mode $\delta\left(\mathrm{v}\right.$, discharge, $\left.\omega_{t}\right)=\left(\mathrm{e}^{\mathrm{At}} \mathrm{v}\right.$, discharge $)$.

A state event is generated when the threshold, $\mathrm{T}$ is exceeded while charging, $\mathrm{C}(\mathrm{v}$,charge $) \equiv[\mathrm{v}>\mathrm{T}]$ and near zero when discharging $\mathrm{C}(\mathrm{v}$,discharge $) \equiv[\mathrm{v}<-\varepsilon]$. The action taken to switch mode and start recovery: $\mathrm{A}(\mathrm{T}$, charge $)=(-\mathrm{R}$, discharge $)$ ( $\mathrm{R}$ is a parameter for the level to which the recovery drops) 
$\mathrm{A}(-\varepsilon$, discharge $)=(0$, charge $)$ This model separates its input pulses into two classes - those that cause it to fire and those that do not. Thus it can serve as a recognizer for the former pulse segments.

\subsection{Hierarchical Modular Brain Architecture}

As illustrated in Figure 6, the development taken so far leads to a hierarchical modular structure for brain architecture. At the top, we start with cognitive behavior in the form of regular languages that, as discussed in the introduction, can model relatively simple human-to-human interactions such as greetings. We then showed how to realize these behaviors in the form of coupled models of abstract components such as Recognizers and Activation nodes modeled in ISS formalism. In turn, we seek to realize these components by biologically plausible elements such as HISS-modeled spiking neurons. More likely, using morphism constructs (Muzy and Zeigler 2019) the functionality required for these components will come from assemblies of such elements into communities with an architectural constraint organization illustrated at the bottom of Figure 6. Petersen and Sporns (2015) argue that brain networks negotiate an economical tradeoff minimizing physical connection cost and maximizing information exchange that lead to hierarchical modular architecture. This form of structure is consistent with the realization of the abstract components as communities of neural elements and suggests further research into explaining development of such structures. Indeed, Petersen and Sporns (2015) indicate that new approaches are needed for mapping brain networks engaged in specific cognitive tasks that can capture their rapid reconfiguration and dynamic functional connectivity. Computational models of dynamic brain activity are indispensable for understanding the complex patterns of signaling and communication within and between brain networks. Developing such models based on the enhancement of DEVS modeling and simulation with HISS using dynamic structure and workload models (Zeigler and Kim 2019) is therefore on the agenda of future research.

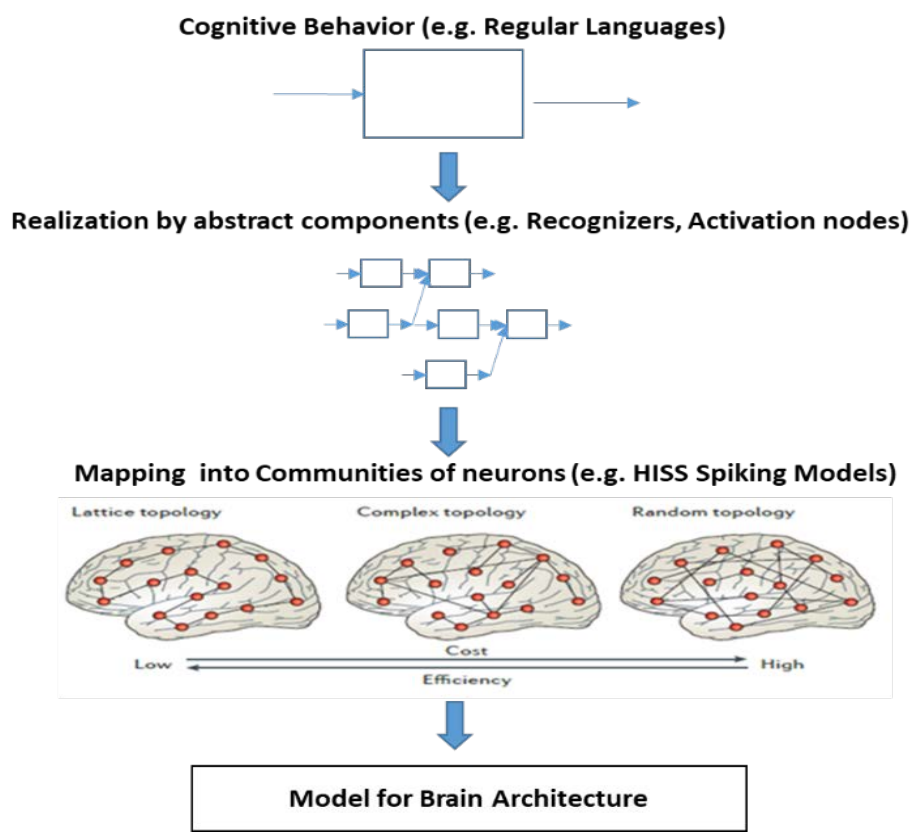

Figure 6: Hierarchical Modular Brain Model.

\section{RELATED RESEARCH}

Kleene (1956) introduced the concept of regular events as the temporal behavior of the finite logic nets defined by McCulloch and Pitts (1943). McNaughton and Yamada (1960) were the first to present algorithms for converting automata state graphs to behaviorally equivalent regular expressions and conversely. Since then, regular expressions have been extensively studied for convenient specification of fast string searches and similar applications (see e.g. Bispo et al 2006). Asarin et al (2001) defined timed 
regular expressions, a formalism for specifying discrete behaviors augmented with timing information, and prove that its expressive power is equivalent to the Timed Automata of Alur and Dill (1994). They also presented an algebraic framework for timed behaviors, time-event sequences and piecewise-constant signals. Châne et al (2004) explored the relation between timed automata and DEVS. Hwang (2014) developed a class hierarchy among DEVS and its variants in terms of sets of event trajectories leading to an expressiveness hierarchy of DEVS, FDDEVS, Timed Automata, and Clock-based DEVS. Ciatto et al (2017) developed a model of spiking neurons in Timed Automata Networks and provided a language and it's encoding into Timed Automata to model patterns of spikes and pauses. Seck et al (2004) developed DEVS models of human behavior under stressful conditions. None of these works focus on construction of general systems, particularly, hybrid ISS simulation coupled models that realize regular expressions intended to close the gap between cognitive behavior and neural circuits. Further they do not make the connection between the resulting hierarchical modular architectures and similarly structured models existing within and between brain networks.

\section{CONCLUSIONS}

We have shown how hybrid iterative systems specification (HISS) offers an abstraction that supports development of hybrid cyber-physical models. To show this we proved that given a temporally represented finite alphabet and a regular expression over the alphabet, we can construct an iterative systems specification coupled model that constitutes a networked recognizer for the regular expression. Based on this construction, we discussed how to close the gap between cognitive behavior and neural circuits. The form of structure we developed is consistent with the realization of the HISS components as communities of neural elements and suggests further research on, and development of, such structures. Neuroscientists have noted that such computational models of dynamic brain activity are needed to understand the complex patterns of signaling and communication within and between brain networks. This paper has set the foundation for future development of such models based on the enhancement of DEVS modeling and simulation with hybrid iterative systems specifications. Further research is needed to establish the neurocognitive plausibility of HISSs that are eventually implemented over the DEVS framework.

\section{REFERENCES}

Alur R. and D.L. Dill. 1994. "A theory of timed automata". Theoretical Computer Science, vol. 126,pp.183-235.

Asarin E., Caspi, P., and Maler, O. 2001.Timed Regular Expressions, https://www.irif.fr/ $\sim$ asarin/papers/revised.pdf._Accessed Dec. 15, 2019.

Bispo J., I. Sourdis, J. Cardoso, and Vassiliadis, R. 2006. "Regular expression matching for reconfigurable packet inspection". In IEEE International Conference on Field Programmable

Technology. pp. 119-126. Bangkok, Thailand.

Carandini, M. 2012. "From Circuits to Behavior: A Bridge Too Far?”. Nature Neuroscience, vol. 15(4), pp. 507-509.

Castro S., Mosterman, P. J. Akshay, Rajhans, H. and Valenti, R. 2019. "Challenges in the Operation and Design of Intelligent Cyber-Physical Systems" in Complexity Challenges in Cyber Physical Systems: Using Modeling and Simulation (M\&S) to Support Intelligence, Adaptation and Autonomy, edited by S, Mittal and A. Tolk pp. 1-25 .New York, Wiley.

Ciatto, G., De Maria, E., Di Giusto. C. 2017. Modeling Third Generation Neural Networks as Timed Automata and verifying their behavior through Temporal Logic. Research Report, Université Cöte d'Azur, CNR,S 13S, France.. 41a1-0147394b.

Châne, F., Giambiasi, N., Paillet, J. 2004." From DEVS Model to Timed Automata".Software Engineering Research and Practice. pp 498-504. 
Hwang M. Ho, 2014 “Taxonomy of DEVS variants”. DEVS '14: Proceedings of the Symposium on Theory of Modeling \& Simulation. Pages 1-6. Tampa, FL

Kleene, S.C. 1956."Representation of Events in Nerve Nets and Finite Automata", Automata Studies, Edited by C.E. Shannon and J. McCarthy, pp. 23-46, Princeton U. Press.

Makin, S. 2016. "Deciphering the Language of the Brain". Scientific American, published online January 31. 2016. https://www.scientificamerican.com/article/deciphering-the-language/, Accessed $15^{\text {th }}$ Sept 2019.

McCullogh W.S and W.H. Pitts 1943. "A Logical Calculus of Ideas Immanent in Nervous Activity”.Bulletin of Mathematical Biophysics, Vol. 5, pp.115-133,

\section{AUTHOR BIOGRAPHIES}

BERNARD P. ZEIGLER is Professor Emeritus of Electrical and Computer Engineering at the University of Arizona, Tucson, AZ, USA and Chief Scientist of RTSync Corp., Phoenix, AZ, USA. Dr. Zeigler is a Fellow of IEEE and SCS and received the INFORMS Lifetime Achievement Award. He is a co-director of the Arizona Center of Integrative Modeling and Simulation. His e-mail address is zeigler@rtsync.com. 